\title{
Properties of galactic $\mathrm{B}[\mathrm{e}]$ supergiants. I. Cl Camelopardalis
}

\author{
A. S. Miroshnichenko ${ }^{1,2}$, V. G. Klochkova ${ }^{3,4}$, K. S. Bjorkman ${ }^{1}$, and V. E. Panchuk ${ }^{3,4}$ \\ ${ }^{1}$ Ritter Observatory, Dept. of Physics \& Astronomy, University of Toledo, Toledo, OH 43606-3390, USA \\ 2 Central Astronomical Observatory of the Russian Academy of Sciences at Pulkovo, 196140, Saint-Petersburg, Russia \\ ${ }^{3}$ Special Astrophysical Observatory of the Russian Academy of Sciences, Karachai-Cirkassian Republic, Nizhnij Arkhyz, \\ 369167, Russia \\ ${ }^{4}$ Isaac Newton Institute of Chile, SAO Branch
}

Received 10 April 2002 / Accepted 23 May 2002

\begin{abstract}
We present a study of the high-resolution $(R=60000)$ optical spectrum of the $\mathrm{B}[\mathrm{e}]$ supergiant CI Cam obtained 4 years after its all-wavelength outburst. The profiles of most of the emission lines show a triple-peaked structure, an effect previously not observed. The $\mathrm{Na}$ I $\mathrm{D}$-lines clearly have 2 interstellar absorption components, suggesting that the system is most probably located within the Perseus arm at a distance of $\leq 3 \mathrm{kpc}$. Uncertainties of the distance toward the object, its luminosity, and physical parameters of the circumstellar disk are discussed. Simple observational tests are suggested to clarify these issues.
\end{abstract}

Key words. stars: emission-line, Be - stars: individual: CI Cam - techniques: spectroscopic

\section{Introduction}

Peculiar Be or $\mathrm{B}[\mathrm{e}]$ stars were distinguished from other earlytype emission-line stars in the Milky Way nearly 30 years ago by Allen $\&$ Swings $(1972,1976)$, who found a correlation between the presence of forbidden emission lines and near-IR excess. These authors selected 65 objects with the above properties and suggested 3 main hypotheses to explain this behaviour: formation of planetary nebulae as a single event, interaction of an OB star with a late-type companion, and direct ejection from a particularly massive Be or Oe star. Follow up studies showed that all the scenarios worked, although they cannot explain the nature of all $\mathrm{B}[\mathrm{e}]$ stars. For example, $7 \mathrm{~B}[\mathrm{e}]$ objects were later recognized as pre-main-sequence intermediate-mass stars (Lamers et al. 1998).

In the mid 1980's a population of B[e] supergiants, which share the basic properties of the galactic $\mathrm{B}[\mathrm{e}]$ stars in addition to a high luminosity, was discovered by Zickgraf et al. (1985) in Magellanic Clouds. These objects show hybrid spectra with evidence for a fast and hot stellar wind in the UV region and a slow and cooler wind in the optical region. These properties were explained by the presence of a circumstellar (CS) disk. In particular, Bjorkman (1998) showed that carbon-rich dust can be formed in the outer regions of such disks.

Eleven members of the galactic $\mathrm{B}[\mathrm{e}]$ group were classified as high-luminosity stars. The most well-known examples include an LBV $\eta$ Carinae, an LBV candidate HDE 316285 (Hillier et al. 1998), and a pre-WR star HDE 326823 (Sterken et al. 1995). This classification still remains controversial for some of the objects mainly due to the difficulties of distance

Send offprint requests to: A. S. Miroshnichenko,

e-mail: anatoly@physics.utoledo.edu determination in the Galaxy. For example, MWC 300 was classified as a B[e] star (Allen \& Swings 1976); as a Herbig Ae/Be star by Finkenzeller \& Mundt (1984) because of the presence of a dark nebula around it; and as a hypergiant by Wolf \& Stahl (1985) due to the presence of fluorescent Fe III emission lines, sensitive to the luminosity.

MWC 84, also known as CI Cam, was considered an unclassified B[e] star (Lamers et al. 1998) until it experienced a strong outburst on 1998 March 31 (e.g., Frontera et al. 1998), which was detected in all wavelength regions from $\gamma$-ray to radio. Detection of the outburst at high energies raised a question about the existence of a compact companion (a neutron star or a black hole) near the visible star. Since the outburst, CI Cam has been extensively monitored at different wavelengths and with different techniques. Recent monitoring results have been reported by Orlandini et al. (2000), Clark et al. (2000), and Barsukova et al. (2002).

One of the most important results of recent studies of CI Cam is that it was suggested to be a high-luminosity star, and thus became the only galactic $\mathrm{B}[\mathrm{e}]$ supergiant with a possible degenerate companion. However, despite good progress in revealing its nature, several problems remain unsolved. They include uncertain distance $(D)$ and luminosity, binary orbital parameters, and the CS disk properties. For example, $D$ ranges from 1 to $\geq 5 \mathrm{kpc}$ in different papers. Robinson, et al. (2002; hereafter R02) recently attempted to address these problems using high-resolution spectroscopy (resolving power $R \sim 60000$ ) of CI Cam obtained 15-17 days after the outburst. At that time the spectral lines were still enhanced, compared to the quiescent state of the object, and might have affected the derived physical parameters. We obtained spectroscopic observations with a similar resolution almost 4 years after the outburst. 
The line profiles in our spectra turned out to be remarkably different from those reported by R02 and those appeared in earlier lower-resolution data. Using the results of our observations, we address the mentioned problems, suggest observational tests toward their solution, and show the importance of high-resolution spectroscopy to improve our knowledge about this unique object.

\section{Observations and data reduction}

The observations were obtained at the 6-m telescope of the Special Astrophysical Observatory (SAO) of the Russian Academy of Sciences with the échelle-spectrograph NES, mounted in the Nasmyth-2 focus and equipped with a $2 \mathrm{~K} \times$ 2 K CCD-chip (Panchuk et al. 1999). This setup allowed us to achieve $R \sim 60000$. The spectra were obtained on 2002 January 25 and February 4 in the spectral ranges 3800-5000 and 4600-6050 A, respectively.

The cosmic ray traces were removed by median averaging of two subsequent spectra. A hollow cathode Th-Ar lamp was used for the wavelength calibration. The first steps of the data reduction process (cosmic ray trace removal, background subtraction, and spectral orders extraction) were done under the ECHELLE context of MIDAS (version 01FEB), while the final steps (normalization to the continuum level and radial velocity $(R V)$ and equivalent width measurements) were completed using $\operatorname{IRAF}^{1}$.

\section{Spectral line characteristics}

Despite the object's popularity, high-resolution spectroscopic observations of it are still rare. In addition to the spectra obtained by R02, observations with a resolution better than $1 \AA$ were obtained on fewer than 10 nights out of a set of about a thousand of exposures since the outburst time (Hynes et al. 2002; hereafter H02). No high-resolution spectra obtained before the outburst have been reported so far. At low and medium resolution, the emission line profiles look single-peaked and narrow (e.g., Jaschek \& Andrillat 2000). The spectrum is dominated by lines of $\mathrm{H} \mathrm{I}, \mathrm{He}$, and Fe II. Some of the latter are blended. No photospheric lines have been detected.

The line profile structure becomes resolved at $R \sim 60000$, and it is different close to the outburst time and much later. R02 reported an almost rectangular profile shape of singly ionized metal lines with flat or double-peaked tops. The double-peaked structure is present only at a level of $\sim 5 \%$ from the line tops, except for a few [Fe II] lines where the central depression is a bit deeper. In our spectra, most of the weak and intermediatestrength lines have triple-peaked profiles with a blueshifted central peak, so that the blue and central peaks are close to each other in both intensity and velocity (Fig. 1). The depression between the red and central peak is significant, reaching half of the line intensity in the strongest lines. At the same time,

${ }^{1}$ IRAF is distributed by the National Optical Astronomy Observatories, which are operated by the Association of Universities for Research in Astronomy, Inc., under contract with the National Science Foundation. very strong lines, such as He I and Balmer lines, have mostly double- or single-peaked profiles (Fig. 2). In fact, these profiles may also be triple-peaked with a broader central peak, which blends with the blue one or even with both the blue and red peak. This transition can be noticed in a sequence of the Fe II line profiles of different strength, shown in Fig. 3.

We detected essentially the same emission lines as those reported by R02, except for those of $\mathrm{Fe}$ I. R02 mentioned that they found only the Fe I lines of Mult. 15 and showed one of them (their Fig. 7) at $15429 \AA$, which is not the strongest line in the Multiplet. We also see an extremely weak line at this wavelength (Fig. 1). However its $R V$ is consistent with the others, if we assume that it is a Fe III line $\lambda 5430.14 \AA$ (Coluzzi 1993). No other members of the Fe I Mult. 15, which contains 12 lines in a range from 5269 to $5506 \AA$, were detected in our spectrum. Thus, we assume that the Fe III line was also present in the R02 spectra, instead of the $\mathrm{Fe}_{\mathrm{I}}$ line. This suggestion is supported by the detection of $\mathrm{He}$ II emission lines in the spectra, obtained only near the outburst. Their presence indicates that the excitation temperature of the emission-line formation region was higher at that time.

We measured $R V \mathrm{~s}$ by means of two methods. The first method calculates the centroid $R V$ of the whole profile, while the second one gives the bisector $R V$ based on the line wings (essentially we matched original and mirrored profiles in the velocity space). The methods give slightly different results, because of the previously mentioned profile asymmetry. The blueshifted central peak makes the centroid $R V$ s more negative than the bisector $R V \mathrm{~s}$ with an average shift of $-1.0 \pm 0.3 \mathrm{~km} \mathrm{~s}^{-1}$. Since the line wings are almost vertical and the central peak is narrow, we consider the bysector method more accurate in determining the systemic $R V$. The mean heliocentric $R V$, derived this way, turned out to be $-47.1 \pm 0.8 \mathrm{~km} \mathrm{~s}^{-1}$ (20 intermediate intensity lines mainly of $\mathrm{Fe}$ II were used). This result is within the measurement errors of R02 $\left(-44.4 \pm 0.6 \mathrm{~km} \mathrm{~s}^{-1}\right)$. The strong lines have more negative $R V \mathrm{~s}$ (between -50 and $-55 \mathrm{~km} \mathrm{~s}^{-1}$ ), most likely due to the effect of a blueshifted central peak.

The only absorption features we detected are diffuse interstellar bands (DIB) at 5705, 5780, 5797, and $5850 \AA$ and the $\mathrm{Na}$ I D-lines at 5889 and $5895 \AA$. The DIB equivalent widths are consistent with those reported earlier by H02. The Na I Dline profiles consist of an emission component and 2 absorption components. In our spectrum, the emission components are significantly weaker than near the outburst. Their intensities, normalized to the underlying continuum, are 1.6 and 1.4 versus $\sim 9$ and $\sim 7$ (H02) for $\mathrm{D}_{2}$ and $\mathrm{D}_{1}$, respectively (Fig. 4). The absorption components are located at the heliocentric $R V \mathrm{~s}$ of -3.5 and $-35.5 \mathrm{~km} \mathrm{~s}^{-1}$. There is no trace of the third absorption component, suspected by $\mathrm{H} 02$, at $-45 \mathrm{~km} \mathrm{~s}^{-1}$. The D-line profile extension to more negative $R V \mathrm{~s}$ near the outburst might be due to CS matter, moving toward us. No photospheric lines were detected in our spectra.

\section{Discussion}

The results of our observations, obtained during the quiescent state of CI Cam, allow us to discuss several important issues, mentioned in Sect. 1, which are not clear yet. 


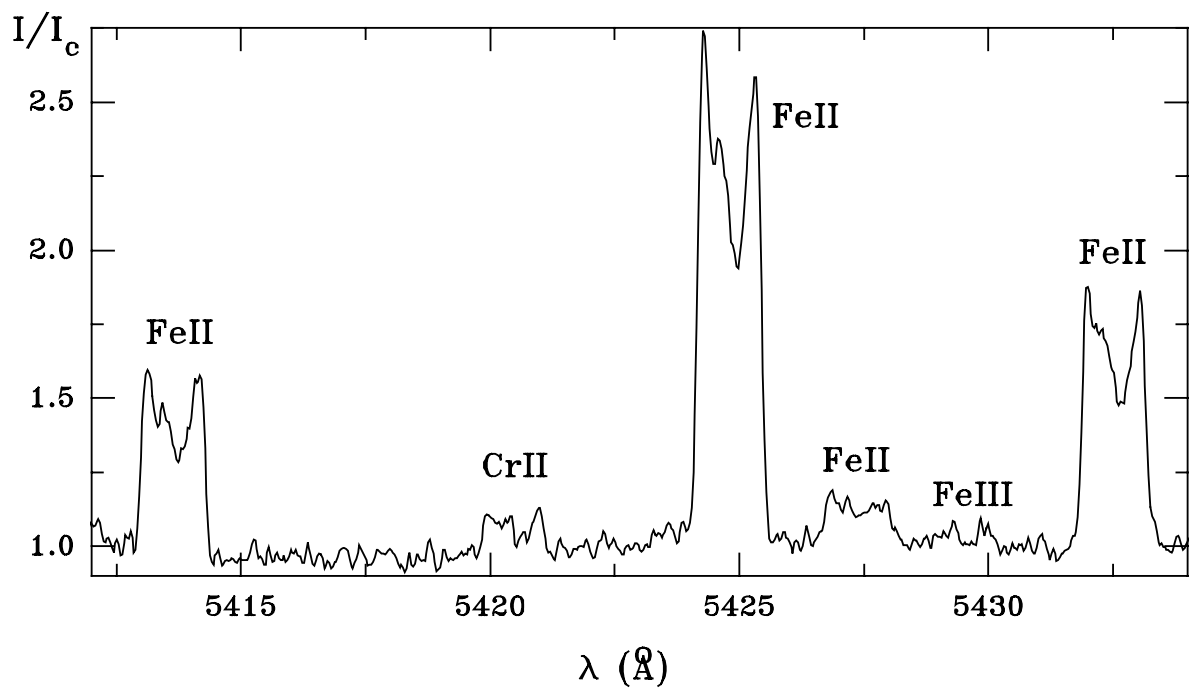

Fig. 1. Metallic lines in the spectrum of CI Cam of 2002 February 4. The intensities are in continuum units, and the wavelengths are in $\AA$.
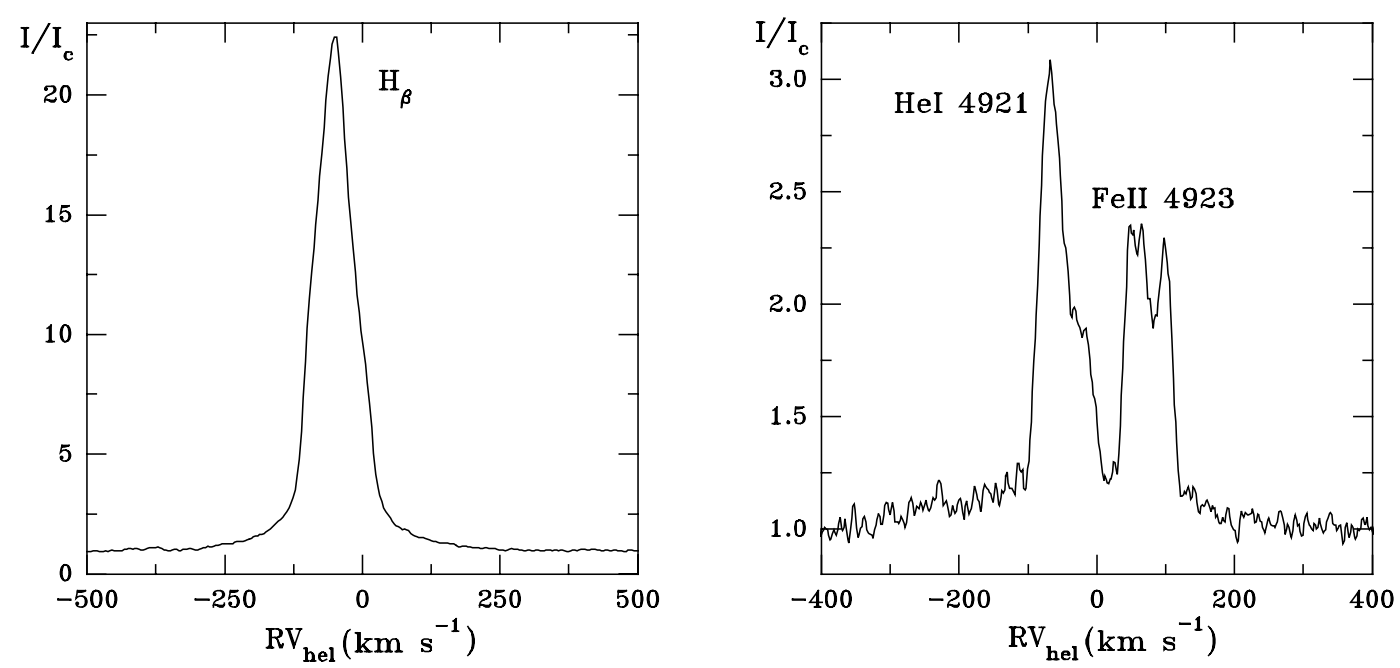

Fig. 2. Examples of the Balmer and He I lines in the spectrum of CI Cam of 2002 February 4. The intensities are in continuum units, and the heliocentric $R V \mathrm{~s}$ are in $\mathrm{km} \mathrm{s}^{-1}$.

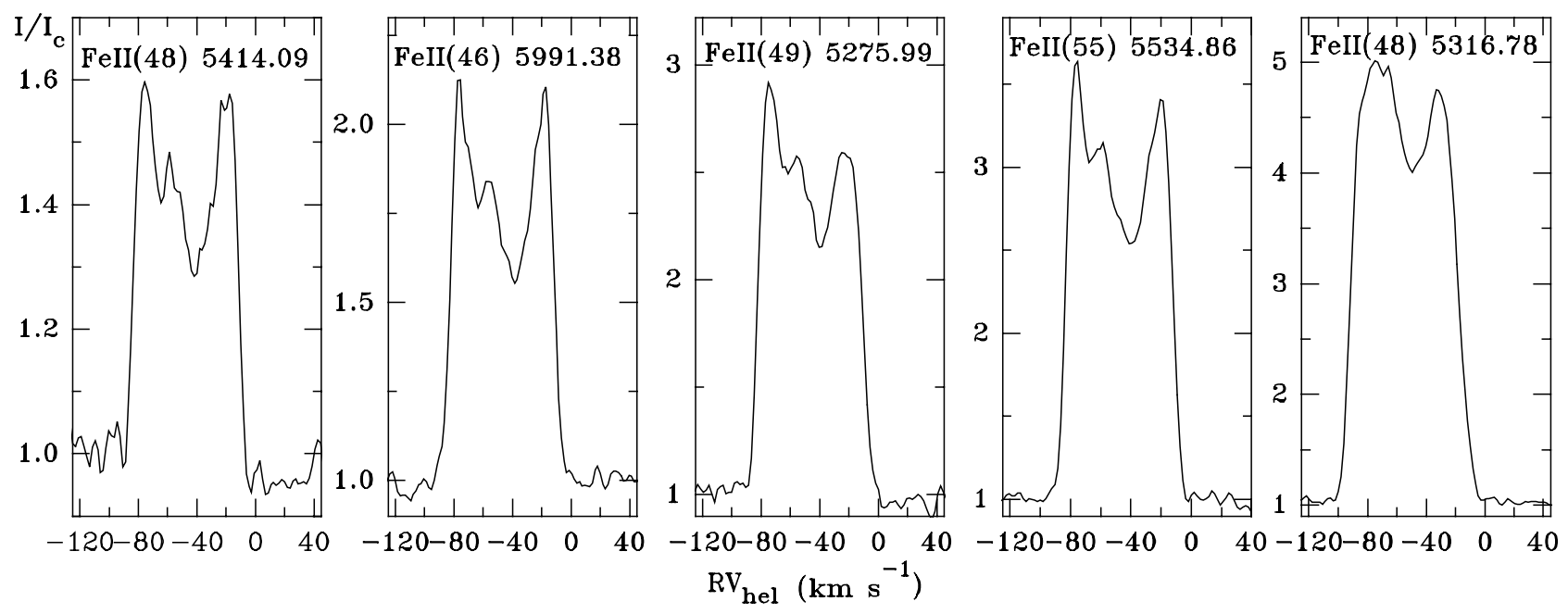

Fig. 3. Examples of the Fe II lines in the spectrum of CI Cam of 2002 February 4. The intensities are in continuum units, and the heliocentric $R V \mathrm{~s}$ are in $\mathrm{km} \mathrm{s}^{-1}$. 


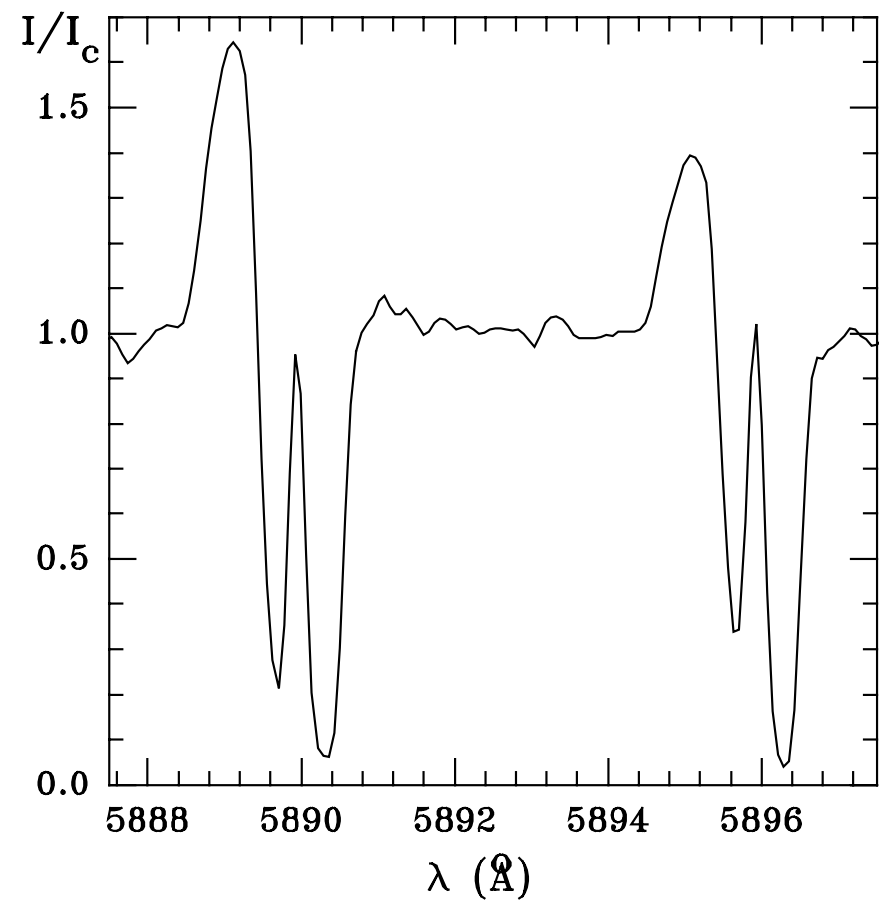

Fig. 4. Na I D-lines in the spectrum of CI Cam of 2002 February 4. The intensities are in continuum units, and the wavelengths are in $\AA$.

\subsection{Distance and luminosity}

The spectral and photometric peculiarities rule out the possibility of applying the spectral type and luminosity criteria for normal stars to CI Cam. As $\mathrm{HO2}$ mentioned, the presence of $\mathrm{He}$ I lines in emission along with the absence of He II lines (except for the short period after the outburst) suggest a spectral type of B1-2 for the visible star. The presence of CS matter makes the interstellar extinction estimate uncertain. The most reliable interstellar extinction estimate, $E_{B-V}=0.85 \pm 0.05 \mathrm{mag}$ and $A_{V}=2.3 \pm 0.3 \mathrm{mag}$, seems to be derived from the UV absorption feature at $2175 \AA$ (R02). Applying this colour-excess to the mean colour-index $B-V \sim 0.9$ mag observed in quiescence (Bergner et al. 1995; Barsukova et al. 2002), one can calculate a dereddened $B-V \sim 0.05 \mathrm{mag}$. This is significantly redder than expected for an extinction-free early B-type star $(B-V \sim-0.25$ mag, e.g., Wegner 1994), thus confirming the presence of a CS effect on the object's spectral energy distribution.

The CS emission in the form of free-free and bound-free radiation seems to be rather strong, since the photospheric lines are not seen even at $R \sim 60000$. Its amount depends on the CS matter distribution. In highly accelerated winds of supergiants this continuum emission is negligible due to a very fast density decrease with distance from the stellar surface. In classical Be stars, which are thought to be surrounded by Keplerian disks (e.g., Hummell 2000), the density gradient is shallower, resulting in an additional CS emission up to $0.3 \mathrm{mag}$ in the $V$-band (Waters et al. 1987). In CI Cam, the emission lines are much stronger and narrower than in classical Be stars, probably implying that the CS emission is stronger too. Thus the visible star in the CI Cam system is most likely intrinsically fainter than it appears. This is a potential source of uncertainty in the luminosity determination for the object. The CS extinction is probably small, because the CS dust does not seem to lie on the line of sight, due to the disk inclination (see Sect. 4.2) and a dust-free high-temperature zone near the star.

The distance toward CI Cam also remains uncertain. The relationship between the interstellar extinction and $D$ is poorly known in this direction, as was already noted by $\mathrm{R} 02$ and $\mathrm{H} 02$. A better way to infer $D$ is by measuring the systemic $R V$. Since no photospheric lines are seen, the emission lines tracing the CS matter distribution around the star are usually used (e.g., Humphreys et al. 1989). This method is reasonable for CI Cam, as its emission lines are very narrow and have well-defined, almost vertical, wings.

As we mentioned above, different authors estimated it from 1 to $\geq 5 \mathrm{kpc}$. For example, R02 claim $D \geq 5 \mathrm{kpc}$, while $\mathrm{H} 02$ consider $D \sim 4-5 \mathrm{kpc}$ mainly from a kinematical consideration using galactic rotation curves, based on the observations of interstellar gas. Additionally, in the Na I D-line profiles H02 found 2 strong absorption components and a trace of the third one, at a $R V \sim-45 \mathrm{~km} \mathrm{~s}^{-1}$, which might suggest that CI Cam is located beyond the Perseus spiral arm at $D=6-8 \mathrm{kpc}$. At the same time, if we apply a method based on the kinematics of late-type supergiants by Dubath, Mayor, \& Burki (1988), the mean heliocentric $R V$ for the Fe II lines, $-45 \mathrm{~km} \mathrm{~s}^{-1}$ (an average of R02 and our results), gives a $D=2.2 \mathrm{kpc}$. These authors studied the $R V$ distribution of 272 stars with absolute values $\leq 35 \mathrm{~km} \mathrm{~s}^{-1}$ within $\sim 2.5 \mathrm{kpc}$ from the Sun. However, it seems to work for more distant stars with larger $R V \mathrm{~s}$ as well. The distances toward such peculiar objects as AG Car (LBV, $D=6 \pm 1 \mathrm{kpc}$, Humphreys et al. 1989) and MWC 314 (LBV candidate, $D \sim 3 \mathrm{kpc}$, Miroshnichenko et al. 1998), estimated by means of this method, are consistent with those from other methods.

The above $D$ places CI Cam inside the Perseus arm, whose outer edge is considered to be at a $D=2.5-3 \mathrm{kpc}$ (Kimeswenger \& Weinberger 1989). These authors presented evidence for the existence of an outer arm in the second galactic quadrant $\left(90^{\circ} \leq l \leq 180^{\circ}\right)$. This arm is traced by all usual indicators of the spiral structure, such as open clusters, OB associations, $\mathrm{H}$ II regions, and luminous stars (which are located up to $b=+9^{\circ}$ ). It is natural to expect that it also contains interstellar matter, which would imprint its signature in the $\mathrm{Na}$ I D-line profiles as at least one more blueshifted absorption component.

In contrast, we detected only 2 such components, which belong to the local and Perseus arm, in the spectrum of CI Cam. From this consideration, we suggest that CI Cam is located not farther than $3 \mathrm{kpc}$ from the Sun, corresponding to a luminosity $\log L / L_{\odot} \leq 4$. Here we assumed no CS contribution to the object's brightness, $V=11.6$ mag (Barsukova et al. 2002), $A_{V}=2.3 \mathrm{mag}$ (R02), and a bolometric correction of $-2 \mathrm{mag}$ (Miroshnichenko 1997). The visible star would be even less luminous, when the CS continuum emission (which can be as large as $\sim 1 \mathrm{mag}$, assuming a simple model like that of Waters et al. 1987) is taken into account. Nevertheless, all the kinematic methods depend on the Galaxy model and on the galactocentric distance of the Sun in particular. 
One way to reconcile this discrepancy is to locate and take spectra of early-type supergiants at $D \geq 3 \mathrm{kpc}$, positionally close to CI Cam, in the Na I D-lines region. Such stars would have optical brightnesses of $5 \mathrm{mag} \leq V \leq 10 \mathrm{mag}$, assuming an absolute visual magnitude of $-7 \mathrm{mag} \leq M_{V} \leq-5 \mathrm{mag}$ (Strajzhys \& Kuriliene 1981) and $3 \mathrm{mag} \leq A_{V} \leq 5 \mathrm{mag}$. If an outer arm does exist, the third absorption component of the $\mathrm{Na}$ I D-lines would be seen at $R \geq 20000$. Several relatively bright candidates with such parameters (e.g., $\mathrm{BD}+55^{\circ} 837$, HDE 237213, LS $\mathrm{I}+56^{\circ} 92$ ) can be found in Miroshnichenko et al. (2000). This method is based on luminosity calibration for normal stars, which can be determined spectroscopically using photospheric lines in the yellow region (see Miroshnichenko et al. 1998).

We note here that the properties of CI Cam, such as strong emission-line spectrum and steep decrease of the IR flux longward of $\sim 10 \mu \mathrm{m}$, make it similar to a group of $\mathrm{B}[\mathrm{e}]$ stars, tentatively called Be stars with warm dust (see Miroshnichenko et al. 2002 for a review). Only a few of the 19 group members have luminosities higher than $\log L / L_{\odot}=4$, and there is growing evidence that these objects are binary systems. One of them, AS 78, is located in only $3^{\circ}$ from CI Cam, has a similar heliocentric $R V\left(-41 \mathrm{~km} \mathrm{~s}^{-1}\right)$, and the same structure of the $\mathrm{Na}$ I D-lines (Miroshnichenko et al. 2000). We estimated its kinematical $D \sim 2.5 \mathrm{kpc}$, which corresponds to a luminosity $\log L / L_{\odot}=3.9 \pm 0.1$. Among these objects, CI Cam is still unique because of its outburst. Nevertheless, investigation of this group may also help to understand CI Cam.

\subsection{Disk properties}

As discussed above, the CS matter can contribute significantly to the object's brightness. Let us consider its properties in more detail. The triple-peaked structure of most of the emission lines, in combination with the lack of absorption components even in the strongest ones (such as $\mathrm{H} \alpha$ ), is consistent with the presence of a disk, inclined at an intermediate angle with respect to the line of sight. The profile structure would not be resolved if the disk is oriented pole-on, while a much deeper central depression or even a P Cyg-type absorption (depending on the disk thickness) would appear in the line profiles if the disk is edgeon. This conclusion does not agree with that of $\mathrm{H} 02$, who argue that the disk is seen pole-on, simply because the line profiles in their spectra were not resolved or were contaminated by the outburst ejection.

The profile blue and red peaks are most likely formed in the disk's densest parts, close to the star, similarly to those of classical Be stars (e.g., Hummell 2000). The central peak seems to be formed in a lower velocity, but also dense, region far away from the star. This region may be a ring at the Roche lobe boundary or a stream around the first Lagrangian point, through which the matter is transfered toward the invisible companion. In the latter case, we may be able to see $R V$ variations of the central peak due to the orbital motion. The whole profile's $R V$ may also vary with the orbital phase, like in classical Be binaries (e.g., Harmanec et al. 2000). It is hard to estimate the orbital period, since we do not know much about the secondary and the disk size. Perhaps regular spectroscopic observations of CI Cam at high-resolution could help to find the former and, hence, to constrain the latter.

A detailed discussion of the disk physical parameters is beyond the scope of this paper. We only comment on a few issues which, in our opinion, need to be further investigated. First, the disk is most likely not as cool (electron temperature $T_{\mathrm{e}} \sim 7000 \mathrm{~K}$ ) as R02 suggested. As we mentioned above (Sect. 3), Fe I lines are not present in our spectra and probably were not present right after the outburst either. Since He I lines are always present in emission and the disk seems to be compact (e.g., narrow line profiles, lack of cold dust), its temperature is most likely high in the regions of line formation.

Second, R02 argue that the disk region, where hydrogen and helium lines are formed, has a low density $\left(n_{\mathrm{e}} \leq 10^{7} \mathrm{~cm}^{-3}\right)$. This conclusion is based on the intensity ratio of 2 weak He I lines ( $\lambda 4713$ and $\lambda 5048 \AA$ ), modelled with unreported CS parameters. R02 also mention that this ratio may have substantial errors. In our spectrum, these lines are clearly resolved and have equivalent widths of 1.4 and $0.6 \AA$, respectively. This leads to an intensity ratio of $\sim 3$ (not far from that of R02, $3.8 \pm 0.4$ ), which was estimated taking into account the continuum intensity ratio at the corresponding wavelengths using a Kurucz (1994) model for a star with an effective temperature of $25000 \mathrm{~K}$. Yet this result is not accurate, because we do not know the amount of the CS emission. Thus, the suggestion that the disk has a low density is not well-established. Moreover, if the density is low, we would probably see photospheric lines. Therefore, the disk density can be much higher $\left(n_{\mathrm{e}} \sim 10^{10}\right.$ $10^{12} \mathrm{~cm}^{-3}$ ), which is consistent with the Balmer line strengths and Balmer decrement (R02). This suggestion is also supported by the presence of a very few [Fe II] emission lines in our spectra. From this fact R02 estimated $n_{\mathrm{e}} \sim 10^{10} \mathrm{~cm}^{-3}$ for the region of the iron lines formation. Application of the selfabsorption curve method (Friedjung 1998) to the observed Fe II lines shows that all the lines, even those with equivalent widths of $\sim 0.1 \AA$, are optically thick. In fact, consistency of the density estimates for the hydrogen and iron lines might imply that both originate in the same region, making the envelope model simpler than that (a slow, mostly spherical wind, where the iron lines are formed and a faster wind, where the hydrogen and helium lines are formed) considered by R02. The full-width at half-maximum of the emission lines is similar for all the mentioned species, while broader wings of the Balmer lines can be a result of electron scattering.

Third, R02 calculate the size of the CS dust region (13 $\leq$ $r_{\text {shell }} \leq 52 \mathrm{AU}$ which corresponds to 2.6 to 10.4 mas at $D=$ $5 \mathrm{kpc}$ ) using an energy balance equation for an optically thin spherical envelope. They also refer to an observed angular diameter of CI Cam (5.2 mas in the $K$-band) which seems to be consistent with their calculations. However, the disk can be optically thick in the continuum (see above), which may reduce the dust condensation radius due to attenuation of the direct stellar radiation. Furthermore, since the fast, less dense wind is seen in the UV (R02), the dust is most likely formed only in the outer parts of the disk. Therefore, the spherical approach would give wrong results for the dust region size. The observed angular size at $2 \mu \mathrm{m}$ refers to the hottest $\left(T_{\mathrm{d}} \sim 1000 \mathrm{~K}\right)$ dust 
around CI Cam, corresponding to $15 \mathrm{AU}$ at $D=3 \mathrm{kpc}$. From the IRAS flux ratios $\left(F_{12} / F_{25}=2.8\right.$ and $\left.F_{25} / F_{60}=3.8\right)$ one can conclude that the system lacks cold dust with $T_{\mathrm{d}} \sim 150-$ $200 \mathrm{~K}$. Hence, the dust region seems to be compact. If we assume that the quoted size roughly corresponds to the Roche lobe size of the visible component, it would give an orbital period estimate of about 10 years for a reasonable assumption about the system's total mass $\left(\sim 20 M_{\odot}\right)$. On the other hand, the dusty disk could be circumbinary, which would make the period estimate meaningless. The orbit may also have a high eccentricity, which might have caused the outburst during a periastron passage in 1998 and allowed the disk to grow as the distance between the companions increases. A similar process was recently observed in an eccentric $(e=0.95)$ binary system $\delta$ Sco (Miroshnichenko et al. 2001), where the density of the gaseous disk increased for a short period near periastron and then dropped significantly with evidence for disk growth. In CI Cam, the near-IR flux is still above the pre-outburst level (Clark et al. 2000), while the optical fluxes returned back to this level in a few weeks after the outburst. The latter also supports our suggestion that the dust is distributed in the disk, whose plane does not pass through the line of sight. In any case, a 2D modelling of the dusty envelope around CI Cam in combination with high-resolution imaging in the IR is necessary to constrain the system model.

\section{Conclusions}

We obtained and analysed high-resolution optical spectra of CI Cam, a binary system containing a $\mathrm{B}$ [e] star and an invisible compact object (neutron star or black hole). The observations were taken almost 3 years after its outburst. The emission line profiles are clearly resolved and show a triple-peaked structure in most cases, suggesting an intermediate inclination angle of the CS disk. No photospheric lines have been detected. The $\mathrm{Na}$ I D-lines show only 2 interstellar absorption components, suggesting the object's location within the Perseus spiral arm.

Our attention was focused on unsolved problems, which hamper understanding of this rather unique object. These problems include uncertainties in the distance and luminosity of the system, binary orbital parameters, and conditions in the CS disk. We suggest observational tests to clarify these issues. In particular, spectroscopy of supergiants, located farther than $\sim 3 \mathrm{kpc}$ from the Sun in the direction of CI Cam will verify the presence of an observable amount of interstellar matter beyond the Perseus arm. Regular high-resolution spectroscopy of the star once every few days may put constraints on the system orbital period. Contemporaneous photometric observations are also very desirable to help in calibrating the line intensity ratios and study further evolution of the CS dust. High-resolution IR imaging can provide information about spatial distribution of the CS dust in the system.

Acknowledgements. A. M. and K. S. B. acknowledge support from NASA grant NAG5-8054. Karen Bjorkman is a Cottrell Scholar of the Research Corporation, and gratefully acknowledges their support. V.K. acknowledges support of the Russian Foundation for Basic Research (grant RFBR-02-02-16085). This work was supported in part by the U.S. Civilian Research \& Development Foundation
(CRDF) grant RP1-2264. This research has made use of the SIMBAD database operated at CDS, Strasbourg, France.

\section{References}

Allen, D. A., \& Swings, J.-P. 1972, Astrophys. Lett., 10, 83

Allen, D. A., \& Swings, J.-P. 1976, A\&A, 47, 293

Barsukova, E. A., Borisov, N. V., Goranskij, V. P., Lyuty, V. M., \& Metlova, N. V. 2002, Astron. Rep., 46, 275

Bergner, Yu. K., Miroshnichenko, A. S., Yudin, R. V., et al. 1995, A\&AS, 112, 221

Bjorkman, J. E. 1998, in B[e] stars, ed. C. Jaschek, \& A.-M. Hubert, Ap\&SS Library, 233, 189

Clark, J. S., Miroshnichenko, A. S., Larionov, V. M., et al. 2000, A\&A, 356,50

Coluzzi, R. 1993, Bull. Inf. CDS, 43, 7

Dubath, P., Mayor, M., \& Burki, G. 1988, A\&A, 205, 77

Finkenzeller, U., \& Mundt, R. 1984, A\&AS, 55, 109

Friendjung, M. 1998, in B[e] stars, ed. C. Jaschek, \& A.-M. Hubert, Ap\&SS Library, 233, 79

Frontera, F., Orlandini, M., Amati, L., et al. 1998, A\&A, 339, L69

Harmanec, P., Habuda, P., Štefl, S., et al. 2000, A\&A, 364, L85

Hillier, D. J., Crowther, P. A., Najarro, F., \& Fullerton, A. W. 1998, A\&A, 340, 483

Hummell, W. 2000, in Be Phenomenon in Early-Type Stars, ed. M. A. Smith, H. F. Henrichs, \& J. Fabregat, ASP Conf. Ser., 214, 396

Humphreys, R. M., Lamers, H. J. G. L. M., Hoekzema, N., \& Cassatella, A. 1989, A\&A, 218, L17

Hynes, R. I., Clark, J. S., Barsukova, E. A., et al. 2002, A\&A, in press

Jaschek, C., \& Andrillat, Y. 2000, in Be Phenomenon in Early-Type Stars, ed. M. A. Smith, H. F. Henrichs, \& J. Fabregat, ASP Conf. Ser., 214, 83

Kimeswenger, S., \& Weinberger, R. 1989, A\&A, 209, 51

Kurucz, R. L. 1994, Smithsonian Astrophys. Obs., CD-ROM No. 19

Lamers, H. J. G. L. M., Zickgraf, F.-J., de Winter, D., Houziaux, L., \& Zorec, J. 1998, A\&A, 340, 117

Miroshnichenko, A. S. 1997, in Fundamental Stellar Properties: The Interaction Between Observation and Theory, Proc., ed. T. R. Bedding (Publ. School of Physics, University of Sydney, Australia), IAU Symp., 189, 50

Miroshnichenko, A. S., Frémat, Y., Houziaux, L., et al. 1998, A\&AS, 131,469

Miroshnichenko, A. S., Chentsov, E. L., Klochkova, V. G., et al. 2000, A\&AS, 147, 5

Miroshnichenko, A. S., Fabregat, J., Bjorkman, K. S., et al. 2001, A\&A, 377, 485

Miroshnichenko, A. S., Bjorkman, K. S., Chentsov, E. L., \& Klochkova, V. G. 2002, in Exotic Stars as Challenges to Evolution, ed. C. A. Tout, \& W. Van Hamme, Proc. IAU Coll., 187, ASP Conf. Ser., in press

Orlandini, M., Parmar, A. N., Frontera, F., et al. 2000, A\&A, 356, 163

Panchuk, V. E., Klochkova, V. G., \& Najdenov, I. D. 1999, Preprint of the Special Astrophys. Observ., No. 135

Robinson, E. L., Ivans, I. I., \& Welsh, W. F. 2002, ApJ, 565, 1169

Sterken, C., Stahl, O., Wolf, B., Szeifert, T., \& Jones, A. 1995, A\&A, 303, 766

Strajzhys, V., \& Kuriliene, G. 1981, Ap\&SS, 80, 353

Waters, L. B. F. M., Coté, J., \& Lamers, H. J. G. L. M. 1987, A\&A, 185,206

Wegner, W. 1994, MNRAS, 270, 229

Wolf, B., \& Stahl, O. 1985, A\&A, 148, 412

Zickgraf, F.-J., Wolf, B., Stahl, O., Leitherer, C., \& Klare, G. 1985, A\&A, 143, 421 